
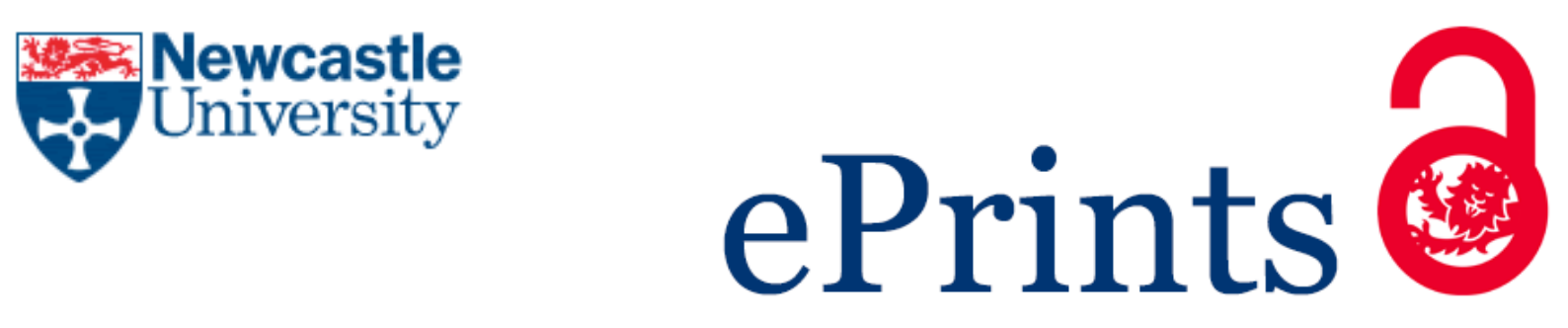

McGeoghegan L, Muirhead CR, Almoosawi S.

Association between an anti-inflammatory and anti-oxidant dietary pattern and diabetes in British adults: Results from the National Diet and Nutrition

Survey rolling programme Years 1-4.

International Journal of Food Sciences \& Nutrition 2016, 67(5), 553-561.

\title{
Copyright:
}

This is an Accepted Manuscript of an article published by Taylor \& Francis in International Journal of Food Sciences \& Nutrition on 04/05/2016, available online:

http://www.tandfonline.com/10.1080/09637486.2016.1179268

DOI link to article:

http://dx.doi.org/10.1080/09637486.2016.1179268

Date deposited:

$22 / 04 / 2016$

Embargo release date:

04 May 2017

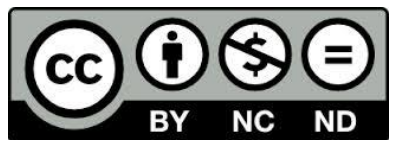

This work is licensed under a

Creative Commons Attribution-NonCommercial-NoDerivatives 4.0 International licence 


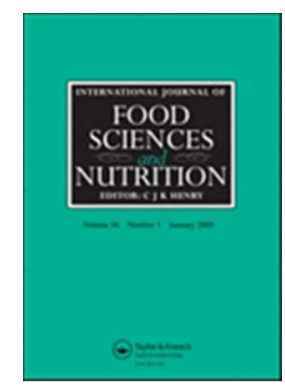

\section{Association between an anti-inflammatory and anti-oxidant dietary pattern and diabetes in British adults: Results from the National Diet and Nutrition Survey rolling programme Years 1-4}

\begin{tabular}{|r|l|}
\hline Journal: & International Journal of Food Sciences and Nutrition \\
\hline Manuscript ID & CIJF-2015-1096.R2 \\
\hline Manuscript Type: & Research Paper \\
\hline Date Submitted by the Author: & 08-Apr-2016 \\
\hline Complete List of Authors: & $\begin{array}{l}\text { almoosawi, suzana; Newcastle University, Human Nutrition Research } \\
\text { Centre and Institute of Health \& Society, } \\
\text { Muirhead, Colin; Newcastle University, Human Nutrition Research Centre } \\
\text { and Institute of Health \& Society } \\
\text { McGeoghegan, Louise; Newcastle University, Faculty of Medical Sciences }\end{array}$ \\
\hline Keywords: & $\begin{array}{l}\text { Glycosylated Haemoglobin, Nutritional epidemiology, Inflammation, } \\
\text { Antioxidant, Diet, type-2 diabetes, Dietary patterns, Cross-sectional survey }\end{array}$ \\
\hline
\end{tabular}

\section{SCHOLARONE \\ Manuscripts}


1 Association between an anti-inflammatory and anti-oxidant dietary pattern and

2 diabetes in British adults: Results from the National Diet and Nutrition Survey rolling

3 programme Years 1-4

4

5 L McGeoghegan ${ }^{1}$, C R Muirhead and S Almoosawi $^{3}$ *

6

$7{ }^{1}$ Faculty of Medical Sciences, Newcastle University; ${ }^{2}$ Institute of Health \& Society,

8 Newcastle University ; Human Nutrition Research Centre and Institute of Health \& Society,

9 Newcastle University

10

$11 *$ Corresponding author \& reprints:

12 suzana.almoosawi@newcastle.ac.uk, Human Nutrition Research Centre and Institute of 13 Health \& Society, Newcastle University, Newcastle upon Tyne, NE2 4HH, United Kingdom; 14 tel +441912227648

15

16 Abstract word count: 150

17 Word count: 3561

18 Number of tables: 4

19 Number of figures: 1

20

21

22

23

24 Short Running Title: Dietary patterns and diabetes

25 


\begin{abstract}
26 Abstract
27 This study investigated the cross-sectional association between an anti-inflammatory and 28 anti-oxidant dietary pattern and diabetes in the National Diet and Nutrition Survey rolling 29 programme Years 1-4. A total of 1531 survey members provided dietary data. Reduced Rank 30 Regression was used to derive an anti-inflammatory and anti-oxidant dietary pattern. Serum 31 C-reactive protein and plasma carotenoids were selected as response variables and markers of 32 inflammation and antioxidant status, respectively. Overall, 52 survey members had diabetes. 33 The derived anti-inflammatory and anti-oxidant dietary pattern was inversely related to C34 reactive protein and positively to carotenoids. It was associated with lower odds of diabetes 35 (multivariate adjusted OR for highest compared with lowest quintile: 0.17; 95\%CI: 0.04-0.73; 36 $P$ for linear trend=0.013). In conclusion, an anti-inflammatory and anti-oxidant dietary 37 pattern is inversely related to diabetes. Further research is required to understand the overall 38 framework within which foods and nutrients interact to affect metabolic pathways related to 39 diabetes risk.
\end{abstract}


41 Keywords: Glycosylated Haemoglobin; C-reactive protein; carotenoids; dietary patterns;

42 National Diet and Nutrition Survey; Diet; Nutritional epidemiology

43 Abbreviations: National Diet and Nutrition Survey NDNS; Reduced Rank Regression RRR;

44 C-reactive protein CRP; Odds Ratio OR; Lower reference nutrient intake LRNI; Diet In

45 Nutrients Out (DINO); Glycosylated haemoglobin $\mathrm{Hb}_{\mathrm{Alc}}$; Computer Assisted Personal

46 Interview CAPI; National Statistics Socio-economic Classification NSSEC

47

48 
49 INTRODUCTION

50 Inflammation and oxidative stress play a central role in the pathophysiology of type 2 51 diabetes (Rosen et al. 2001;Spranger et al. 2003). However, to date, little is known of how 52 various dietary components interact to influence this inflammatory and anti-oxidant process 53 and subsequently the development of type 2 diabetes. Most current research has focused on 54 assessing individual nutrients or foods in relation to diabetes risk. Such research ignores the 55 complexity of eating behaviour and diet providing an oversimplified view of the role of 56 nutrition in chronic disease prevention (Sievenpiper and Dworatzek 2013). Although 57 globally the role of diet as a whole is increasingly recognised in the nutritional management 58 of diabetes, the same is not true for prevention (Sievenpiper and Dworatzek 2013). Of the 59 few studies that have investigated the relationships between dietary patterns and diabetes, 60 most assessed diet using food frequency questionnaires, a method that captures data on usual 61 intake and remains useful in assessing dietary intake in large cohorts, but which also suffers 62 from systematic bias due to errors in estimating portion size and limitations concerning the 63 food groups included in the questionnaires (Schulze et al. 2005;Nettleton et al. 64 2007; Nettleton et al. 2008). Because foods are consumed in combination, it is imperative for 65 observational studies to utilise more advanced methods of analysis to investigate diet-disease 66 relationships. Reduced rank regression (RRR) is more effective than the widely-used 67 principal component analysis, since it permits the use of prior knowledge to identify dietary 68 patterns that explain as much variation as possible in a set of response variables presumed to 69 be related to disease risk (Hoffmann et al. 2004;Hoffmann et al. 2005;Michels and Schulze 70 2005;Schulze and Hoffmann 2006;DiBello et al. 2008). The resulting dietary patterns thus 71 provide information on potential biological pathways that link diet to disease (Michels and 72 Schulze 2005). To our knowledge, only two studies have so far utilised this method to 73 identify dietary patterns related to biomarkers linked to diabetes (Heidemann et al. 
74 2005;Schulze et al. 2005). Of these studies, none has been conducted in the UK or utilised 75 more robust methods of dietary assessment such as food diaries.

76

77 sectional survey of a nationally representative sample of the UK population (Whitton et al.

78 2011). It utilises food diaries to collect data on food consumption, nutrient intakes and 79 nutritional status. This method of dietary assessment is known to correlate more highly with 80 biomarker data [11] and to be less prone to measurement error than food frequency 81 questionnaires (Heidemann et al. 2005; Stephen 2007). The NDNS rolling programme also 82 contains up to date information on lifestyle and blood biomarkers in people aged 1.5 years 83 and above living in England, Wales, Scotland and Northern Ireland, thereby providing a 84 unique opportunity for studying the association between diet and diabetes in a nationally 85 representative sample.

86 The present study investigated the association between an anti-inflammatory and anti87 oxidant dietary pattern derived using reduced rank regression (Hoffmann et al. 88 2004;Hoffmann et al. 2005;Schulze and Hoffmann 2006) and diabetes status in the National 89 Diet and Nutrition Survey (NDNS) rolling programme Years 1-4.

\section{METHODS}

\section{Study population}

93 The survey design and sampling methods of NDNS have been described extensively in 94 previous studies (Bates et al. 2014). The NDNS rolling programme is a nationally95 representative survey of food consumption, nutrient intakes and nutritional status in people 96 aged 1.5 years and above living in England, Wales, Scotland and Northern Ireland (Bates et 97 al. 2014). The survey is implemented by a consortium of three organisations: the National 98 Centre for Social Research (NatCen), MRC Human Nutrition Research, and the Department 
99 of Epidemiology and Public Health at the University College London Medical School.

100 Fieldwork for the NDNS rolling programme began in 2007 and is on-going.

101 The overall response rate for fully productive individuals (i.e. those completing three

102 or four dietary recording days) was 56\% in Year 1, 57\% in Year 2, 53\% in Year 3 and 55\% in

103 Year 4, giving a sample size of 3,450 fully-productive adults aged 19 years and over (Bates et

104 al. 2014). All individuals who completed three or four days of dietary recording were eligible

105 for a nurse visit. Of these, 1531 adult survey members (19-65 years) went on to have a nurse

106 visit

107 This survey was conducted according to the guidelines set in the Declaration of 108 Helsinki. All procedures involving human volunteers were approved by the Oxfordshire 109 Research Ethics Committee and all participants gave informed consent for each phase of the 110 study.

\section{Dietary assessment}

113 Details of dietary assessment have been provided elsewhere (Riley 2010;Department of 114 Health 2011; Whitton et al. 2011). Diet was assessed using 4 day estimated food diaries. In

115 Year 1, the recording period started on a Thursday, Friday or Saturday and included both 116 weekend days. This led to an over-representation of weekend days while Wednesdays were 117 never represented. From Year 2 onwards, the food diary recording period was changed to 118 allow all days of the week to be equally represented. Participants were asked to report all 119 foods and drinks consumed both at home and away from home. Portion sizes were estimated 120 using household measures, weights on labels or by comparison with life-size photographs of 121 spoons and glasses. To improve compliance and recording, food diaries were reviewed by 122 trained interviewers on the second day of recording and participants were asked to clarify any 123 uncertainties (Bates et al. 2014). When completed, diaries were checked by interviewers with 
124 respondents and missing details added to improve completeness. Diet diaries were coded and 125 edited using DINO (Diet In Nutrients Out) and nutrient intakes calculated using the NDNS nutrient databank (Whitton et al. 2011).

127

128

129

130

131

132

133

134

135

136

137

138

139

140

141

142

143

144

145

146

147

\section{Definition of diabetes and biomarkers of glycaemic control}

Fasting blood samples were collected for measurements of glycosylated haemoglobin $\left(\mathrm{Hb}_{\mathrm{A} 1 \mathrm{c}}\right)$ at four stages of recruitment between February 2008 to March 2009, April 2009 to May 2010, April 2010 to August 2011, and April 2011 to August 2012 (Bates et al. 2014). For $\mathrm{Hb}_{\mathrm{A} 1 \mathrm{c}}$ analysis, samples were sent by nurses overnight to Addenbrooke's Hospital in Cambridge. Consistency of measurements was checked by running three controls on each run. Optimal concentration of $\mathrm{Hb}_{\mathrm{A} 1 \mathrm{c}}$ was defined as $\mathrm{Hb}_{\mathrm{Alc}}<6.5 \%$, while survey members with $\mathrm{Hb}_{\mathrm{Alc}}$ values $\geq 6.5 \%$ were deemed to have diabetes. Two categories of diabetes status were defined: no diabetes, and self-reported or undiagnosed diabetes. This categorisation was based on self-reported diabetes status and $\mathrm{Hb}_{\mathrm{Alc}}$ concentrations. Survey members were deemed to have no diabetes if they did not report having diabetes and if $\mathrm{Hb}_{\mathrm{Alc}}$ was normal. Survey members who reported having diabetes but had normal or high $\mathrm{Hb}_{\mathrm{Alc}}$ were deemed to have self-reported diabetes. Survey members who did not report having diabetes but had high $\mathrm{Hb}_{\mathrm{Alc}}$ were considered to have undiagnosed diabetes. Self-reported diabetes was assessed by asking participants the following questions: Do you have any long-standing illness, disability or infirmity? By long-standing I mean anything that has troubled you over a period of time, or that is likely to affect you over a period of time? The variables for longstanding illness had 42 codes. Participants who were assigned the code "diabetes including hyperglycaemia" were deemed to have self-reported diabetes. 


\section{Additional measures and covariates}

149 Height and weight were measured using a portable stadiometer and weighing scales. BMI 150 (weight $(\mathrm{kg}) /$ height $\left(\mathrm{m}^{2}\right)$ ) was classified according to the criteria set by the World Health 151 Organisation and National Institute for Health and Clinical Excellence. A Computer Assisted 152 Personal Interview (CAPI) was also conducted during the initial visit by trained interviewers 153 to obtain information on respondent's health status; smoking and drinking habits; socio154 economic characteristics; medication use and some usual dietary behaviours. Socio-economic 155 status was defined based on the National Statistics Socio-economic Classification (NS-SEC) 156 as: 1) higher occupations, 2) intermediate occupations, 3) lower occupations, and 4) never 157 worked or long-term unemployed.

159 Statistical methods

160 Data were weighed to correct for unequal sample selection, non-response for household and 161 individual interview and non-response to nurse visit (Food Standards Agency. 2010). This 162 weighing factor adjusts for differences in socio-demographic variables, such as age, sex, 163 ethnicity and region, between participants and non-participants to the nurse visit to ensure 164 survey sample is representative of the UK population (Food Standards Agency. 2010).

Differences between survey members with or without diabetes were assessed using 167 independent sample t-tests for continuous variables or $\chi^{2}$ tests for categorical variables. 168 Trends in energy and macronutrient intake across quintiles of dietary pattern scores were 169 assessed using a non-parametric trend test ("nptrend" in Stata), an extension of the Wilcoxon 170 rank-sum test for trend across ordered groups. Bivariate associations were evaluated using 171 chi-square tests for categorical variables. 
173 Reduced Rank Regression

174 To derive dietary patterns, reduced rank regression (RRR) was used on 49 pre-defined food 175 groups. Serum C-reactive protein (CRP) a marker of inflammation, and plasma total 176 carotenoids, a marker of anti-oxidant status, were selected as response variables. These markers were selected on the basis of their relation to diabetes and influence by diet (Ford et al. 2003; Higuchi et al. 2015; Sluijs et al. 2015) . Inflammation is known to reduce cellular anti-oxidant capacity (Khansari et al. 2009), and has been shown to be inversely related to carotenoids have been shown to correlate negatively with CRP and other markers of inflammation (Kritchevsky et al. 2000). Both response variables were skewed and were logtransformed prior to inclusion in RRR. Details of quality control procedures and assays used to measure C-reactive protein and plasma total carotenoids have been published elsewhere (Public Health England and Food Standards Agency 2014). Briefly, CRP was assayed using a Dade Behring Dimension RXL Clinical Chemistry Analyser. The assay method is based on a high sensitivity particle enhanced turbidimetric immunoassay (PETIA) technique, with a detection range $\geq 1.0 \mathrm{mg} / \mathrm{L}$. Carotenoids were measured by HPLC coupled with a photodiode array detector using a method derived from Thurnham et al (Thurnham et al. 1988).

RRR was run using a STATA module that implements the SAS partial least squares procedure. The module was installed using the command "ssc install plssas" (2007). Zscores were assigned to each survey member indicating how closely their dietary intake reflects the obtained dietary pattern. Analysis were repeated using food groups adjusted for energy intake. The resulting factor loadings did not differ from the values obtained using the absolute food group intake. Consequently, only data from model including absolute food group intake are presented. 
198 Logistic Regression

199 Odds ratios (ORs) and associated 95\% confidence intervals (CIs) for quintiles of dietary 200 pattern Z-scores in relation to diabetes status were estimated using multivariable logistic 201 regression models. Model 1 adjusted for age, sex and ethnicity. Model 2 additionally adjusted 202 for energy intake. Model 3 included all covariates in Model 2 as well as BMI. Finally, 203 Model 4 included all of the above covariates in addition to adjusting for waist circumference.

204 These covariates were selected on the basis of their relationship with diet and influence on 205 diabetes status (Wang et al. 2005; Shai et al. 2006). BMI and waist circumference data were 206 missing in $8 \%$ and $28 \%$ of the productive adult sample, respectively. These values were 207 assumed to be missing at random and consequently imputed as continuous variables by using 208 multiple imputation. Thirty imputed data sets were created and fitted by using the "ice" and 209 "mim" packages in Stata (StataCorp LP). Sensitivity analysis were conducted comparing 210 findings from imputed data and complete case analysis. Additional sensitivity analysis 211 including social class, smoking status and physical activity as covariates were conducted.

212 These covariates were subsequently dropped from the final model as they did not add to the 213 predictive power of the model. Tests for linear trend of the association between quintiles of 214 dietary patterns and diabetes status were assessed by assigning the median value of each 215 quintile and modelling the variable as a continuous variable.

216 All statistical analyses were carried out by using Stata Statistical Software version 13. 217 The $P$-values reported were 2 -tailed, and a $P$-value $<0.05$ was considered statistically 218 significant.

\section{RESULTS}

221 Characteristics of the study population are provided in Table 1, while intakes of food groups 222 are provided in Table 2. Of the 1531 individuals included in the RRR analysis, 52 had 
223 diabetes. Survey members with diabetes were older $(\mathrm{P}<0.001)$, and had higher BMI $224(\mathrm{P}<0.001)$ and waist circumference $(\mathrm{P}<0.001)$ compared to survey members without diabetes. 225 There were no differences in total energy intake over the day between survey members with 226 or without diabetes.

Two dietary patterns were derived using RRR. Dietary pattern 1 explained the maximum variation in response variables (13\%) and was inversely related to serum CRP and positively to plasma carotenoids. Correlation coefficients ranged from -0.42 for CRP to 0.90 for plasma carotenoids. This anti-inflammatory and anti-oxidant dietary pattern was characterised by high intake of vegetables and fruit and low intake of chips, sugar and white bread (Figure 1). Dietary pattern 2 explained a smaller variation in food intake and was positively associated with serum CRP and plasma carotenoids. As a result, only Dietary pattern 1 was deemed of interest to the current study.

There were significant associations between the anti-inflammatory and anti-oxidant dietary pattern and sex and ethnicity, with a higher proportion of women and non-white survey members being in the highest quintile of Dietary pattern 1 (Table 3). Energy and macronutrient intake varied across quintiles of Dietary pattern 1. In particular, there was a trend towards increased energy intake, protein (expressed as percentage of energy intake), n-3

240 polyunsaturated fatty acids (expressed as percentage of energy intake), fibre, intrinsic milk 241 sugars and starch across increasing quintiles of Dietary pattern 1. By contrast, there was a 242 trend towards lower carbohydrate intake (expressed as percentage of energy intake), 243 particularly non-milk extrinsic sugars, across quintiles of Dietary pattern 1 . There was also a 244 marginally significant trend towards reduced BMI and waist circumference across quintiles 245 of Dietary pattern 1 (Table 3). 
248 95\% CI: 0.04-0.73; $P$ for linear trend $=0.013$; based on adjustment for age, sex, ethnicity and 249 energy intake) (Table 4). After adjustment for BMI and waist circumference, the association 250 weakened slightly but remained significant (OR for highest compared with lowest quintile: $251 \quad 0.22 ; 95 \%$ CI: 0.05-0.96; $P$ for linear trend= 0.047$)$.

252

253 DISCUSSION

254 Interpretation of main findings

255 The current study identified a dietary pattern that was inversely associated with biomarkers of 256 inflammation and positively with biomarkers of anti-oxidant status. This anti-inflammatory 257 anti-oxidant dietary pattern, characterised by high intake of vegetables and fruit and low 258 intake of chips, sugar and white bread, was associated with lower prevalence of type 2 259 diabetes.

260 Our findings are consistent with previous research on the association between dietary 261 patterns and type 2 diabetes risk. In particular, the dietary pattern identified in our study is 262 comparable to the dietary pattern identified in the Nurses' Health Study (Schulze et al. 2005).

263 In the latter study, the dietary pattern was associated with markers of inflammation and 264 endothelial dysfunction, and was characterised by low intake of vegetables and high intake of 265 sugary beverages. This pattern strongly predicted risk of type 2 diabetes independent of BMI 266 and other diabetes risk factors. In contrast in the current study, the association between the 267 derived dietary pattern and diabetes prevalence was reduced slightly following adjustment for 268 BMI and waist circumference. This may suggest that the relationship between the anti269 inflammatory anti-oxidant dietary pattern and diabetes is mediated by BMI and body fat 270 distribution. The latter is in line with previous studies demonstrating that the association 271 between diet and type 2 diabetes risk could be affected by BMI and body fat distribution For 272 instance, in the EPIC-InterAct study, the association between total protein as well as animal 
273 protein and type 2 diabetes risk was attenuated in men after adjustment for body composition

274 (van Nielen et al. 2014). In the latter study, obesity - in particular, abdominal obesity - was an 275 independent risk factor for type 2 diabetes that strongly correlated with protein intake (van 276 Nielen et al. 2014). In our study, waist circumference was found to decrease across quintiles 277 of the anti-inflammatory anti-oxidant dietary pattern. This may imply that the observed 278 dietary pattern reflects an anti-inflammatory and anti-oxidant dietary pattern related to 279 abdominal obesity, although this would need to be explored further. status is well recognised (Ahluwalia et al. 2013;Barbaresko et al. 2013;Uusitupa and Schwab 2013). This knowledge provides us with a basic understanding as to how various dietary constituents exert anti-inflammatory anti-oxidant effects. In the current study, the observed dietary pattern was characterised by increasing intake of vegetables, fruit, nut and fish, and decreasing intake of chips, sugar and white bread. This, in turn, was reflected in the nutrient composition of the dietary pattern; high intake of protein, omega-3 fatty acids, starch and fibre across the quintiles, and low intake of carbohydrate, non-milk intrinsic sugars and extrinsic milk sugars. These findings are noteworthy as they provide us with insight into specific combinations of food and nutrients that might be relevant to reducing inflammation and improving anti-oxidant status. To date, our knowledge of how foods and nutrients interact together and how these need to be combined to achieve a desired health outcome remains limited. What remains clear, however, is that dietary approaches that take into consideration complex interactions between various dietary components have proved to be more effective compared to dietary interventions focusing on a single food or nutrient (Franco et al. 2004; Shirani et al. 2013). One such example is the DASH diet, which has been shown consistently to improve cardiovascular disease risk factors (Siervo et al. 2014) and improve insulin sensitivity (Shirani et al. 2013). Such evidence implies the need to improve 
298 our understanding as to how foods interact to prevent chronic diseases such as type 2

299 diabetes. In this respect, novel methods such as RRR provide a framework for investigating 300 diet-disease relationships taking into account existing knowledge of disease pathways.

301 Nonetheless, there is a need for developing models that will assist nutrition researchers in 302 interpreting dietary patterns further in order to devise dietary recommendations that could 303 assist in preventing chronic diseases.

304 Two interesting findings of the current study are the relative change in the proportion 305 of energy obtained from protein versus carbohydrates across quintiles of the dietary pattern, 306 and the variation in fat composition across the quintiles. These findings warrant further 307 investigation in other observational studies on dietary patterns and type 2 diabetes. Most 308 current dietary guidelines focus on adjusting the percentage energy intake from carbohydrates 309 and fat, while maintaining energy intake from protein at $15 \%$. In relation to clinical practice, 310 there has been a gradual shift in the nutritional recommendations for patients with diabetes, 311 with most current diabetes societies recommending up to $20 \%$ of energy intake from protein 312 (Sievenpiper and Dworatzek 2013). However, the debate concerning varying energy intake 313 from protein in relation to diabetes risk remains controversial (Afshin et al. 2014). In the 314 European Prospective Investigation into Cancer and Nutrition (EPIC)-NL Study, consuming $3155 \%$ energy from total or animal protein at the expense of 5\% energy from carbohydrates or 316 fat was associated with an increased diabetes risk (Sluijs et al. 2010). Similarly, in the EPIC317 InterAct Case-Cohort Study, high total and animal protein intake was associated with a 318 modest elevated risk of type 2 diabetes (van Nielen et al. 2014). This is in contrast to findings 319 from short-term intervention studies demonstrating a beneficial effect of high-protein diets on 320 weight loss and glycaemic control (Ajala et al. 2013). These discrepancies in findings could 321 be attributed to various factors, including the use of food frequency questionnaires to assess 322 food and nutrient intake. A reductionist approach to nutrition research could also be a key 
323 factor, as research that does not take into consideration the context within which nutrients are

324 consumed, often ignores the complexity of diet and the potential additive and synergetic 325 effects of multiple foods and dietary components (Sievenpiper and Dworatzek 2013).

326 Nutrients are known to interact differently when present as foods, and as such there is a 327 pressing need to combine dietary patterns approaches and nutrient approaches in order to gain 328 a stronger understanding of the role of diet in health and disease.

\section{Strengths and Weakness of the Study}

331 One of the main weaknesses of the current study compared to previous studies is the cross332 sectional design of the study which limits any causal inferences. Moreover, given that only a 333 fraction of the productive sample went on to have nurse visits and that the total number of 334 diabetes cases is relatively small, the extent to which the findings can be generalised is 335 limited, although data were weighed to overcome this limitation and to account for the 336 sampling method and non-responsiveness. The small number of cases also hindered further 337 assessment of differences in dietary pattern between survey members with self-reported and 338 undiagnosed diabetes, which is an important weakness of the current study compared to 339 previous studies. The latter investigation could be of interest as it provides insight into 340 changes in food choices following diagnosis of type 2 diabetes. Despite these weaknesses, it 341 is important to acknowledge that the NDNS rolling programme is the only cross-sectional 342 survey in the UK that utilises robust methods of dietary assessment and which is run on a 343 rolling basis. As such, the NDNS rolling programme provides a unique opportunity to 344 generate hypothesis and investigate diet-disease relationships. Undoubtedly, this points 345 towards the importance of continuing to measure biomarkers of diabetes risk in this survey, 346 as the pseudo-longitudinal design of this study might allow us in the future to improve our 
347 understanding of the association between diet and disease, thereby providing the basis for

348 further research and policy development.

349

\section{CONCLUSION AND IMPLICATIONS}

351 The present study identified an anti-inflammatory anti-oxidant dietary pattern which was 352 related to lower prevalence of diabetes. This dietary pattern was characterised by high intake 353 of vegetables and fruit and low intake of chips, sugar and white bread. This research points 354 towards the importance of improving our understanding of the relation between diet as a 355 whole and chronic disease outcomes such as type 2 diabetes, as opposed to focusing on single 356 food or nutrients. Further research is required to understand the overall framework within 357 which various foods interact to affect metabolic pathways related to diabetes risk. Moreover, 358 evidence from observational studies need to be supported by further intervention studies that 359 can examine the effect of an anti-inflammatory anti-oxidant dietary pattern on biomarkers of 360 glucose metabolism. Finally, more research is required to understand the effect of varying 361 energy intake from the various macronutrients whilst taking into account macronutrient 362 sources and the overall diet. Such research will undoubtedly form the basis for developing 363 public health policies that take into consideration the complex nature of diet, shifting the 364 paradigm towards a more holistic view of nutrition.

366 ACKNOWLEDGMENTS

367 The authors would like to thank the Operations Team at NatCen and all interviewers involved 368 for the collection of data; statisticians Dr Adrian Mander and Mark Chatfield for 369 contributions to the survey design; and Melanie Farron-Wilson and Mary Day at the 370 Department of Health for contributions to food composition issues. We also thank all 371 participants of the survey for providing their dietary information. 
372

373 FINANCIAL SUPPORT

374 The NDNS survey was funded by the Department of Health, the Food Standards Agency

375 and Diabetes UK.

376

377 CONFLICT OF INTEREST

378 The authors declare no conflict of interest.

379

380 AUTHORSHIP

381 LM was responsible for analysing and interpreting data, drafting the article and revising and 382 approving the final draft. CRM supervised the project, assisted with data interpretation, 383 revised, edited and approved the final draft of the manuscript. SA conceptualised the study, 384 assisted in the analyses and data interpretation, supervised the research, wrote and revised the 385 manuscript. 


\section{REFERENCES}

1. Afshin A, Micha R, Khatibzadeh S \& Mozaffarian D 2014. Consumption of nuts and legumes and risk of incident ischemic heart disease, stroke, and diabetes: a systematic review and meta-analysis. Am J Clin Nutr, 100, 278-88.

2. Ahluwalia N, Andreeva VA, Kesse-Guyot E \& Hercberg S 2013. Dietary patterns, inflammation and the metabolic syndrome. Diabetes Metab, 39, 99-110.

3. Ajala O, English P \& Pinkney J 2013. Systematic review and meta-analysis of different dietary approaches to the management of type 2 diabetes. Am J Clin Nutr, 97, 505-16.

4. Appendix P Methods of blood analysis and quality control [Internet]. 2014. London: Public Health England and Food Standards Agency.Public Health England and Food Standards Agency [cited 1st January 2015].Available from: https://www.food.gov.uk/sites/default/files/ndns-appendix-p.pdf

5. Barbaresko J, Koch M, Schulze MB \& Nothlings U 2013. Dietary pattern analysis and biomarkers of low-grade inflammation: a systematic literature review. Nutr Rev, 71, 511-27.

6. Dibello JR, Kraft P, Mcgarvey ST, Goldberg R, Campos H \& Baylin A 2008. Comparison of 3 Methods for Identifying Dietary Patterns Associated With Risk of Disease. American Journal of Epidemiology, 168, 1433-1443.

7. Food Standards Agency. 2010. Appendix B Weighting the NDNS core sample. Available:

http://www.food.gov.uk/multimedia/pdfs/publication/ndns0809appendixb.pdf [Accessed $1^{\text {st }}$ January 2015].

8. Ford ES, Mokdad AH, Giles WH \& Brown DW 2003. The Metabolic Syndrome and Antioxidant Concentrations: Findings From the Third National Health and Nutrition Examination Survey. Diabetes, 52, 2346-2352.

9. Franco OH, Bonneux L, De Laet C, Peeters A, Steyerberg EW \& Mackenbach JP 2004. The Polymeal: a more natural, safer, and probably tastier (than the Polypill) strategy to reduce cardiovascular disease by more than 75\%. BMJ, 329, 1447-50.

10. Heidemann C, Hoffmann K, Spranger J, Klipstein-Grobusch K, Mohlig M, Pfeiffer AF, Boeing H, European Prospective Investigation Into C \& Nutrition --Potsdam Study C 2005. A dietary pattern protective against type 2 diabetes in the European Prospective Investigation into Cancer and Nutrition (EPIC)--Potsdam Study cohort. Diabetologia, 48, 1126-34.

11. Higuchi K, Saito I, Maruyama K, Eguchi E, Mori H, Tanno S, Sakurai S, Kishida T, Nishida W, Osawa H \& Tanigawa T 2015. Associations of serum $\beta$-carotene and retinol concentrations with insulin resistance: The Toon Health Study. Nutrition, 31, 975-980.

12. Hoffmann K, Boeing H, Boffetta P, Nagel G, Orfanos P, Ferrari P \& Bamia C 2005. Comparison of two statistical approaches to predict all-cause mortality by dietary patterns in German elderly subjects. Br J Nutr, 93, 709-16.

13. Hoffmann K, Schulze MB, Schienkiewitz A, Nothlings U \& Boeing H 2004. Application of a new statistical method to derive dietary patterns in nutritional epidemiology. Am J Epidemiol, 159, 935-44.

14. Khansari N, Shakiba Y \& Mahmoudi M 2009. Chronic inflammation and oxidative stress as a major cause of age-related diseases and cancer. Recent Pat Inflamm Allergy Drug Discov, 3, 73-80.

15. Kritchevsky SB, Bush AJ, Pahor M \& Gross MD 2000. Serum carotenoids and markers of inflammation in nonsmokers. Am J Epidemiol, 152, 1065-71. 
16. Michels KB \& Schulze MB 2005. Can dietary patterns help us detect diet-disease associations? Nutrition Research Reviews, 18, 241-248.

17. National diet and nutrition survey. Results from Years 1, 2, 3 and 4 (combined) of the rolling programme (2008/2009 - 2011/2012) [Internet]. 2014. London: Department of Health.Bates B, Lennox A, Prentice A, Bates C, Page P, Nicholson S \& Swan G [cited 1st January 2015].Available from: https://www.gov.uk/government/publications/national-diet-and-nutrition-surveyresults-from-years-1-to-4-combined-of-the-rolling-programme-for-2008-and-2009-to2011-and-2012

18. National Diet and Nutrition Survey Headline results from Years 1 and 2 (combined) of the Rolling Programme (2008/2009 - 2009/10) [Internet]. 2011. London: Department of Health.Department of Health [cited $1^{\text {st }}$ January 2015].Available from: http://www.dh.gov.uk/en/Publicationsandstatistics/Publications/PublicationsStatistics/ DH_128166

19. Nettleton JA, Schulze MB, Jiang R, Jenny NS, Burke GL \& Jacobs DR, Jr. 2008. A priori-defined dietary patterns and markers of cardiovascular disease risk in the MultiEthnic Study of Atherosclerosis (MESA). Am J Clin Nutr, 88, 185-94.

20. Nettleton JA, Steffen LM, Schulze MB, Jenny NS, Barr RG, Bertoni AG \& Jacobs DR, Jr. 2007. Associations between markers of subclinical atherosclerosis and dietary patterns derived by principal components analysis and reduced rank regression in the Multi-Ethnic Study of Atherosclerosis (MESA). Am J Clin Nutr, 85, 1615-25.

21. PLSSAS: Stata module to execute SAS partial least squares procedure [Internet]. 2007. Cambridge: Mander, A.[cited 11th February 2014].Available from: https://ideas.repec.org/c/boc/bocode/s456810.html

22. Riley H 2010. NDNS rolling programme - what do the Year 1 results show? Nutrition Bulletin, 35, 235-239.

23. Rosen P, Nawroth PP, King G, Moller W, Tritschler HJ \& Packer L 2001. The role of oxidative stress in the onset and progression of diabetes and its complications: a summary of a Congress Series sponsored by UNESCO-MCBN, the American Diabetes Association and the German Diabetes Society. Diabetes Metab Res Rev, 17, 189-212.

24. Schulze MB \& Hoffmann K 2006. Methodological approaches to study dietary patterns in relation to risk of coronary heart disease and stroke. Br J Nutr, 95, 860-9.

25. Schulze MB, Hoffmann K, Manson JE, Willett WC, Meigs JB, Weikert C, Heidemann C, Colditz GA \& Hu FB 2005. Dietary pattern, inflammation, and incidence of type 2 diabetes in women. Am J Clin Nutr, 82, 675-84; quiz 714-5.

26. Schweigert FJ 2001. Inflammation-induced changes in the nutritional biomarkers serum retinol and carotenoids. Curr Opin Clin Nutr Metab Care, 4, 477-81.

27. Shai I, Jiang R, Manson JE, Stampfer MJ, Willett WC, Colditz GA \& Hu FB 2006. Ethnicity, obesity, and risk of type 2 diabetes in women: a 20 -year follow-up study. Diabetes Care, 29, 1585-90.

28. Shirani F, Salehi-Abargouei A \& Azadbakht L 2013. Effects of Dietary Approaches to Stop Hypertension (DASH) diet on some risk for developing type 2 diabetes: a systematic review and meta-analysis on controlled clinical trials. Nutrition, 29, 93947.

29. Siervo M, Lara J, Chowdhury S, Ashor A, Oggioni C \& Mathers JC 2014. Effects of the Dietary Approach to Stop Hypertension (DASH) diet on cardiovascular risk factors: a systematic review and meta-analysis. Br J Nutr, 1-15.

30. Sievenpiper JL \& Dworatzek PDN 2013. Food and Dietary Pattern-Based Recommendations: An Emerging Approach to Clinical Practice Guidelines for 
Nutrition Therapy in Diabetes (vol 37, pg 51, 2013). Canadian Journal of Diabetes, 37, 135-135.

31. Sluijs I, Beulens JW, Van Der AD, Spijkerman AM, Grobbee DE \& Van Der Schouw YT 2010. Dietary intake of total, animal, and vegetable protein and risk of type 2 diabetes in the European Prospective Investigation into Cancer and Nutrition (EPIC)NL study. Diabetes Care, 33, 43-8.

32. Sluijs I, Cadier E, Beulens JW, Van Der AD, Spijkerman AM \& Van Der Schouw YT 2015. Dietary intake of carotenoids and risk of type 2 diabetes. Nutr Metab Cardiovasc Dis, 25, 376-81.

33. Spranger J, Kroke A, Mohlig M, Hoffmann K, Bergmann MM, Ristow M, Boeing H \& Pfeiffer AF 2003. Inflammatory cytokines and the risk to develop type 2 diabetes: results of the prospective population-based European Prospective Investigation into Cancer and Nutrition (EPIC)-Potsdam Study. Diabetes, 52, 812-7.

34. Stephen AM 2007. The Case for Diet Diaries in Longitudinal Studies. International Journal of Social Research Methodology, 10, 365-377.

35. Thurnham DI, Smith E \& Flora PS 1988. Concurrent Liquid-Chromatographic Assay of Retinol, Alpha-Tocopherol, Beta-Carotene, Alpha-Carotene, Lycopene, and BetaCryptoxanthin in Plasma, with Tocopherol Acetate as Internal Standard. Clinical Chemistry, 34, 377-381.

36. Uusitupa M \& Schwab U 2013. Diet, inflammation and prediabetes-impact of quality of diet. Can J Diabetes, 37, 327-31.

37. Van Nielen M, Feskens EJ, Mensink M, Sluijs I, Molina E, Amiano P, Ardanaz E, Balkau B, Beulens JW, Boeing H, et al. 2014. Dietary protein intake and incidence of type 2 diabetes in Europe: the EPIC-InterAct Case-Cohort Study. Diabetes Care, 37, 1854-62.

38. Wang YF, Rimm EB, Stampfer MJ, Willett WC \& Hu FB 2005. Comparison of abdominal adiposity and overall obesity in predicting risk of type 2 diabetes among men. American Journal of Clinical Nutrition, 81, 555-563.

39. Whitton C, Nicholson SK, Roberts C, Prynne CJ, Pot GK, Olson A, Fitt E, Cole D, Teucher B, Bates B, et al. 2011. National Diet and Nutrition Survey: UK food consumption and nutrient intakes from the first year of the rolling programme and comparisons with previous surveys. Br J Nutr, 106, 1899-1914. 
Table 1 Characteristics of study population $(\mathrm{n}=1531)$. Data are presented as means and SDs for continuous variables or as counts and percentages for categorical variables.

\begin{tabular}{|c|c|c|c|c|c|c|c|}
\hline & \multicolumn{6}{|c|}{$\underline{\text { Diabetes Status }}$} & \multirow[b]{2}{*}{ P-value* } \\
\hline & & No & & & Yes & & \\
\hline & Unweighted $\mathrm{N}$ & Mean (or count) & SD (or \%) & Unweighted $\mathrm{N}$ & Mean (or count) & SD (or \%) & \\
\hline Dietary pattern z-score & 1479 & 0.04 & 1.05 & 52 & -0.16 & 1.21 & 0.195 \\
\hline Age (years) & 1479 & 42 & 12 & 52 & 51 & 11 & $<0.001$ \\
\hline BMI $\left(\mathrm{kg} / \mathrm{m}^{2}\right)$ & 1369 & 27.7 & 5.5 & 46 & 31.6 & 5.7 & $<0.001$ \\
\hline Waist circumference (cm) & 1054 & 93.1 & 14.6 & 42 & 105.2 & 16.2 & $<0.001$ \\
\hline Total daily energy (kcal) & 1479 & 1828 & 584 & 52 & 1716 & 633 & 0.175 \\
\hline Sex ( $\%$ female $)$ & & 842 & 56.9 & & 28 & 53.9 & 0.659 \\
\hline Ethnicity (\% non-white) & & 148 & 10.0 & & 8 & 15.4 & 0.208 \\
\hline \multicolumn{8}{|l|}{ Smoking } \\
\hline$\%$ ex-regular smoker & & 293 & 19.8 & & 16 & 30.8 & 0.103 \\
\hline$\%$ current smoker & & 387 & 26.2 & & 9 & 17.3 & \\
\hline
\end{tabular}


Table 2 Intake of food groups (grams) and percentage consumers of study population.

Food group

Bacon and ham

Beer, lager, cider and perry

Biscuits

Brown granary and wheat germ bread

Buns, cakes, pastries and fruit pies

Burgers and kebabs

Butter

Cheese

Chicken and turkey dishes

Chips, fried/roast potatoes and potatoes

Coated chicken

confectionery

Crisps and savoury snacks

Dry weight beverages

Eggs and egg dishes

Fruit

Fruit juice

High-fibre breakfast cereals

Ice-cream

Low fat milk

Low fat spread not polyunsaturated

Reduced fat spread not polyunsaturated

Low fat spreads and polyunsaturated fatty acids spreads

Meat pies and pastries

Nuts and seeds

Oily fish and other fish

Other breakfast cereals

Other meats and meat products
N Consumers Mean SD

$(\%)$

\begin{tabular}{|c|c|c|c|}
\hline 1531 & (59) & 15 & 20 \\
\hline 1531 & $(33.6)$ & 199 & 491 \\
\hline 1531 & $(60.9)$ & 13 & 18 \\
\hline 1531 & $(34.2)$ & 14 & 27 \\
\hline 1531 & $(48.7)$ & 17 & 27 \\
\hline 1531 & $(13.1)$ & 6 & 19 \\
\hline 1531 & $(30.4)$ & 3 & 7 \\
\hline 1531 & (61.4) & 29 & 37 \\
\hline 1531 & $(66.5)$ & 58 & 75 \\
\hline 1531 & $(64.3)$ & 41 & 46 \\
\hline 1531 & $(14.4)$ & 6 & 18 \\
\hline 1531 & $(48.1)$ & 10 & 19 \\
\hline 1531 & $(49.3)$ & 7 & 11 \\
\hline 1531 & $(14.5)$ & 2 & 10 \\
\hline 1531 & $(49.2)$ & 20 & 36 \\
\hline 1531 & (76.9) & 91 & 104 \\
\hline 1531 & $(37.6)$ & 52 & 114 \\
\hline 1531 & $(42.7)$ & 19 & 43 \\
\hline 1531 & $(18.2)$ & 5 & 13 \\
\hline 1531 & $(80.5)$ & 117 & 125 \\
\hline 1531 & $(5.3)$ & 0.44 & 2 \\
\hline 1531 & $(46.7)$ & 4.65 & 7 \\
\hline 1531 & $(33.8)$ & 3 & 6 \\
\hline 1531 & $(23.3)$ & 11 & 27 \\
\hline 1531 & $76(18)$ & 2 & 8 \\
\hline 1531 & $(46.7)$ & 28 & 50 \\
\hline 1531 & $(29.3)$ & 6 & 12 \\
\hline 1531 & $(17.3)$ & 7 & 24 \\
\hline
\end{tabular}


Other milk and cream

Other potatoes, potato salad dishes

Pasta, rice and other cereals

Pork and dishes

Puddings

Red meat and dishes

sausages

Savoury sauces, pickles gravies, condiments

Soft drinks low calorie

Soft drinks not low calorie

Soup homemade and retail

Spirits and liqueurs

Sugars preserves and sweet Spreads

Tea, coffee and water

Vegetables

White bread

White fish, coated or fried

Wholemeal bread and other bread

Whole milk

Wine

Yogurt, fromage frais and dairy desserts

$\begin{array}{llll}1531 & (19.3) & 9 & 34 \\ 1531 & (65.2) & 45 & 50 \\ 1531 & (76.6) & 78 & 84 \\ 1531 & (19.9) & 10 & 30 \\ 1531 & (22.1) & 11 & 27 \\ 1531 & (58.3) & 61 & 82 \\ 1531 & (32.6) & 14 & 31 \\ 1531 & (81.1) & 24 & 28 \\ 1531 & (35.9) & 108 & 235 \\ 1531 & (49.3) & 123 & 224 \\ 1531 & (27.8) & 32 & 66 \\ 1531 & (12.5) & 5 & 25 \\ 1531 & (64.4) & 11 & 16 \\ 1531 & (98.3) & 1104 & 627 \\ 1531 & (96.5) & 133 & 103 \\ 1531 & (79.4) & 50 & 49 \\ 1531 & (21.6) & 8 & 18 \\ 1531 & (41.5) & 20 & 35 \\ 1531 & (18.9) & 23 & 73 \\ 1531 & (33.9) & 65 & 137 \\ 1531 & (37.5) & 28 & 49\end{array}$


Figure 1 Factor loading for the anti-inflammatory and anti-oxidant dietary pattern.

1

2

3

4

5

6

7

8

9

10

11

12

13

14

15

16

17

18

19

20

21

22

23

24

25

26

27

28

29

30

31

32

33

34

35

36

37

38

39

40

41

42

43

44

45

46

47

48

49

50

51

52

53

54

55

56

57

58

59

60

URL: http://mc.manuscriptcentral.com/cijf Email: furio.brighenti@unipr.it 
Table 3 Survey member characteristics and macronutrient intake according to quintiles of the anti-inflammatory and anti-oxidant dietary pattern.

\begin{tabular}{|c|c|c|c|c|c|c|c|c|c|c|c|}
\hline & \multicolumn{10}{|c|}{ Quintile of dietary pattern (1=lowest quintile, $5=$ highest) } & \multirow[t]{3}{*}{ P-Value } \\
\hline & \multicolumn{2}{|l|}{1} & \multicolumn{2}{|c|}{2} & \multicolumn{2}{|c|}{3} & \multicolumn{2}{|c|}{4} & \multicolumn{2}{|l|}{5} & \\
\hline & $\begin{array}{c}\text { Mean } \\
(\mathrm{n}=307)\end{array}$ & SD & $\begin{array}{c}\text { Mean } \\
(\mathrm{n}=306)\end{array}$ & SD & $\begin{array}{c}\text { Mean } \\
(\mathrm{n}=306)\end{array}$ & SD & $\begin{array}{c}\text { Mean } \\
(\mathrm{n}=306)\end{array}$ & SD & $\begin{array}{c}\text { Mean } \\
(\mathrm{n}=306)\end{array}$ & SD & \\
\hline Ethnicity (\% non-white) & 2.6 & & 5.6 & & 10.8 & & 12.4 & & 19.6 & & $<0.001$ \\
\hline Age (yrs) & 41.8 & 12.7 & 41.7 & 12.7 & 43.1 & 12.9 & 42.0 & 12.1 & 43.6 & 12.1 & 0.107 \\
\hline Waist circumference $(\mathrm{cm})$ & 67.9 & 45.4 & 69.7 & 45.1 & 64.9 & 44.2 & 66.3 & 43.2 & 64.6 & 44.4 & 0.025 \\
\hline Food energy (kcal) & 1730.2 & 579.4 & 1620.5 & 440.6 & 1691.4 & 454.0 & 1778.6 & 494.5 & 1924.9 & 516.4 & $<0.001$ \\
\hline Protein (\% Energy) & 15.0 & 4.0 & 16.3 & 3.4 & 16.9 & 3.7 & 16.8 & 3.5 & 17.2 & 4.2 & $<0.001$ \\
\hline Fat (\% Energy) & 32.8 & 6.7 & 33.5 & 6.2 & 33.0 & 5.8 & 32.5 & 6.4 & 32.8 & 6.8 & 0.345 \\
\hline Saturated fatty acids (\% Energy) & 12.1 & 3.5 & 12.5 & 3.4 & 12.1 & 3.1 & 11.9 & 3.3 & 11.8 & 3.4 & 0.093 \\
\hline Cis-monounsaturated fatty acids (\% Energy) & 12.1 & 2.8 & 12.1 & 2.6 & 11.9 & 2.6 & 11.8 & 2.7 & 11.8 & 3.1 & 0.053 \\
\hline Carbohydrate (\% Energy) & 46.4 & 7.6 & 45.4 & 7.8 & 45.6 & 7.2 & 45.6 & 7.9 & 44.7 & 7.7 & 0.008 \\
\hline Starch (g) & 127.5 & 47.4 & 120.9 & 35.7 & 120.1 & 39.7 & 130.3 & 41.2 & 140.1 & 43.1 & $<0.001$ \\
\hline Total sugars $(\mathrm{g})$ & 104.6 & 53.2 & 87.4 & 44.1 & 94.8 & 40.4 & 96.8 & 44.3 & 98.1 & 37.5 & 0.297 \\
\hline Nonmilkextrinsicsugars (g) & 74.6 & 48.9 & 55.1 & 40.2 & 55.2 & 34.6 & 56.8 & 37.1 & 52.2 & 30.8 & $<0.001$ \\
\hline Intrinsicandmilksugars (g) & 30.0 & 17.1 & 32.3 & 15.1 & 39.6 & 16.4 & 40.0 & 17.9 & 45.9 & 19.6 & $<0.001$ \\
\hline Fibre (g) & 11.6 & 4.4 & 12.3 & 4.0 & 13.6 & 3.7 & 14.8 & 4.7 & 17.3 & 5.0 & $<0.001$ \\
\hline
\end{tabular}


Table 4 Associations between quintiles of the anti-inflammatory and anti-oxidant dietary pattern and OR (95\% CI) of diabetes* .

\begin{tabular}{|c|c|c|c|c|c|c|}
\hline & 1 & 2 & 3 & 4 & 5 & P-value for linear trend \\
\hline Model 1‡ & 1.0 (Ref) & $0.66(0.21,2.08)$ & $0.55(0.16,1.93)$ & $0.5(0.18,1.4)$ & $0.16(0.04,0.69)$ & 0.008 \\
\hline Model 2 & 1.0 (Ref) & $0.66(0.21,2.06)$ & $0.56(0.16,1.94)$ & $0.52(0.19,1.48)$ & $0.17(0.04,0.76)$ & 0.014 \\
\hline Model 3 & $1.0(\operatorname{Ref})$ & $0.74(0.23,2.36)$ & $0.69(0.2,2.41)$ & $0.64(0.22,1.87)$ & $0.21(0.05,0.92)$ & 0.033 \\
\hline Model 4 & 1.0 (Ref) & $0.69(0.22,2.12)$ & $0.69(0.19,2.48)$ & $0.69(0.24,1.98)$ & $0.22(0.05,0.96)$ & 0.047 \\
\hline
\end{tabular}




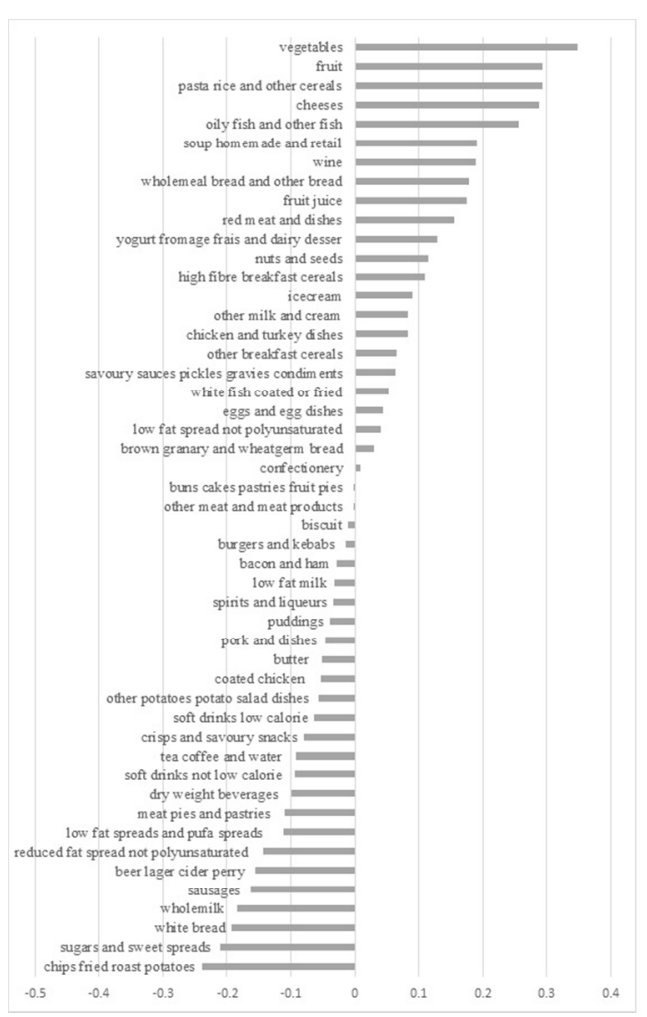

Factor loading for the anti-inflammatory and anti-oxidant dietary pattern. $304 \times 232 \mathrm{~mm}(96 \times 96$ DPI $)$

URL: http://mc.manuscriptcentral.com/cijf Email: furio.brighenti@unipr.it 